\title{
Simulation of VANET routing using A-star Algorithm
}

\section{Reema}

Assistant Professor, Department of

CSE, Sat Kabir Institute of

Technology \& Management,

Bahadurgarh, Haryana, India

\section{Monika}

M. Tech Scholar, Department of

CSE, Sat Kabir Institute of

Technology \& Management,

Bahadurgarh, Haryana, India
Shabnam Kumari

Assistant Professor, Department of

CSE, Sat Kabir Institute of

Technology \& Management,

Bahadurgarh, Haryana, India

\section{ABSTRACT}

VANETs are anticipated to prop a colossal spectrum of mobile distributed applications whose scope is from traffic alert dissemination and dynamic route planning to context-aware advertisement and file sharing. The performance of established routing protocols in VANET is very deprived. The main problem with these protocols is their Route Instability in VANET's. The established node-centric view of the routes (i.e., an established path is a fixed succession of nodes between the source and the destination) leads to frequent broken paths in the presence of VANETs' elevated mobility, Consequently, countless packets are dropped, and the overhead due to path repairs or failure notifications considerably increases, leading to low delivery ratios and elevated transmission delays.

KEYWORD: Quality of Service (QoS), MANET, Route Instability, VANETs

\section{INTRODUCTION}

It is a network without any central control or an "infrastructure-less" network. This type of network is a collection of two or more devices outfitted with wireless communication and networking capability [2].

\subsection{Mobile Ad-Hoc Networks (MANETs)}

The present adoption of the disparate 802.11 wireless standards has caused a dramatic rise in the number of wireless data networks [3]. Nowadays, wireless LANs are highly deployed and the price for wireless equipments is dropping in price. Mobile Ad-hoc Networks (MANETs) is that area which has presently received substantial attention.

MANET is a collection of autonomous mobile nodes that can communicate to every single node in the network via radio waves [4]. There are lots of applications for MANETs that range from small and static low power networks to large and mobile communication systems [5]. One promising application of MANET is the development of VANET [6].

\subsection{Vehicular Ad-Hoc Networks (VANETs)}

At present time cars and other private vehicles are used daily by large number of peoples. The major problem regarding the augmented use of private transport is the growing number of mortalities that occur due to accidents on the roads. Approx 1.2 million people are killed each year in the road accidents. Road traffic safety has been the challenging matter in traffic management. One possible way is to give the traffic information to the vehicles so that they can use it to examine the traffic environment. All the vehicles are movable in nature, hence a mobile network is needed which is self organized and capable of operating without infrastructure support [7].

VANET is an autonomous and self-organized wireless communication network that can be formed by connecting vehicles aiming to upgrade driving security and traffic management with internet access by drivers and programmers [8].

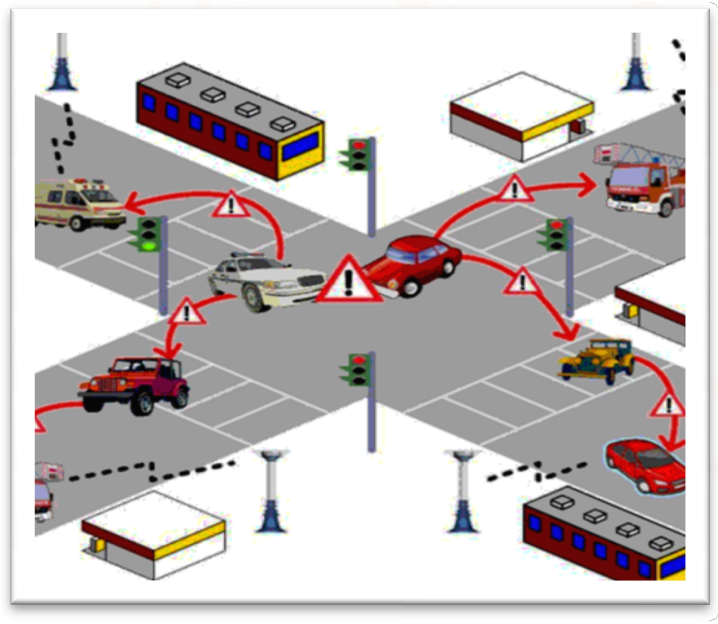

Figure1.1: VANET set up in an urban area 


\section{COMMUNICATION IN VANETS}

There are 2 types of communication possible in VANETS:

- V2V: Vehicles can communicate with other vehicles openly forming vehicle to vehicle communication or inter-vehicle communication.

- V2I: Vehicle can communicate with fixed tools next to the road, called as Road Side Unit (RSU) forming vehicle to infrastructure communication or vehicle to roadside communication.

These types of communications allow vehicles to share different kinds of data, for example, safety data for the purpose of collision prevention, post-accident investigation or traffic jams [9].

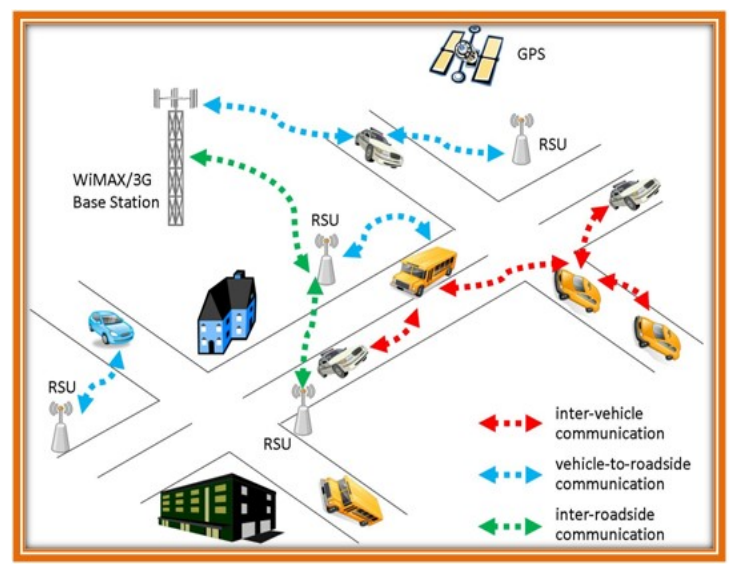

Figure 2.1: Different types of communication in VANETs

\section{WIRELESS ACCESS TECHNOLOGY IN VANETs}

VANET provides a wireless communication between moving vehicles, using a Dedicated Short Range Communication (DSRC). DSRC is IEEE 802.11a revised for low overhead operation to $802.11 \mathrm{p}$, the IEEE then standardizes the entire communication stack by the 1609 family of standards which refers to Wireless Access in Vehicular Environments (WAVE) [9]. DSRC is a $75 \mathrm{MHz}$ licensed spectrum at a 5.9 $\mathrm{GHz}$ band owed by the US Federal Communications Commission (US FCC) in 1999, to be used solely for V2V and V2I communication in the United States. In Europe and Japan the spectrum is allocated at 5.8 $\mathrm{GHz}$ [10]. DSRC/WAVE supports an environment in which vehicles can move up to speed of $200 \mathrm{Kmph}$, covering a contact range of $300 \mathrm{~m}$ and extending up to $1000 \mathrm{~m}$ with a data rate of more than $27 \mathrm{Mbps}$.

\section{VANET ARCHITECTURE}

The communication amid vehicles or between a vehicle and an RSU is achieved through a wireless standard called WAVE. This technique of communication provides a wide range of information to drivers and travelers and allows safety applications to improve road safety and offer a comfortable driving [11]. The main system components are:-

4.1. On Board Unit (OBU) - An OBU is a device usually mounted on-board a vehicle used for exchanging information with RSUs or with other OBUs [11].

4.2. Application Unit (AU) - The AU is the device equipped within the vehicle that utilizes the applications given by the supplier using the communication capabilities of the OBU [11].

4.3. Road Side Unit (RSU) - The RSU is a device normally fixed all along the road side or in crowded locations such as at junctions or near parking spaces [11].

\section{PROBLEM DEFINATION}

VANETs are anticipated to prop a colossal spectrum of mobile distributed applications that range from traffic alert dissemination and dynamic route planning to context-aware advertisement and file sharing. Considering the colossal number of nodes that contribute in these networks and their high mobility, the difficulty yet continue concerning the feasibility of applications that apply end-to-end multi-hop communication in Intersection Routing on City Roads when they are executed in Real-Time Vehicular Traffic Environment.

The main concern is whether the performance of VANET routing protocols can gratify the throughput and delay necessities of such applications. The work focuses on VANET routing in city-based scenarios. Analyses of established routing protocols for MANETs like AODV and DSR demonstrated that their performance in VANETs is very deprived. The main problem with these protocols is their Route Instability in VANET's. The established node-centric view of the routes (i.e., an established path is a fixed succession of nodes between the source and the 
destination) leads to frequent broken paths in the presence of VANETs' elevated mobility, Consequently, countless packets are dropped, and the overhead due to path repairs or failure notifications considerably increases, leading to low delivery ratios and elevated transmission delays.

An enhanced model for intersection based routing on city roads using real-time vehicular traffic for VANET is designed and applied. A-Star Algorithm is used to find optimal path from source to destination. Simulation is done using urban city maps settings in VANET MobiSim which is integrated in NetBeans IDE and the performance will be evaluated and compared with the VTL scheme. The performance of the network will be analyzed on the basis of simulation parameters like packet loss, throughput, average waiting time, average speed etc.

\section{SIMULATION OF VANET APPLICATIONS}

The evaluation of VANET protocols and applications could be made across real outdoor experiments, which are time-costly and claim for a colossal number of resources in order to attain momentous results. Instead, simulation is a far cheaper and easier to use method. Obviously, it directs network and application developers to make use of simulation in order to assess disparate easy or complex and innovative solutions prior to implementing them. In turn, this motivates the interest for the development of simulators that easily incorporate the models and answer to the necessities of VANET applications.

\section{PROPOSED METHODOLOGY}

A-Star Algorithm which is used to traverse graph efficiently is proposed here with some amendments to find optimal path from source to destination. It is a best-first search algorithm which performs better than Dijkstra's algorithm with respect to time.

A-Star makes use of heuristics for decision making. It uses a distance and a cost heuristic function (usually denoted $\mathrm{f}(\mathrm{n})$ ) to find out the arrangement according to which the search will visit the nodes in the Look up Table.

The distance-plus-cost heuristic is the addition of two functions: (i) The path-cost function, which is the cost from the starting node to the current node (denoted by $g(n))$ and

(ii) The admissible "heuristic estimate" of distance to the goal (denoted by $h(n))$.

The A-Star algorithm creates a Look up Table of nodes and maintains two lists, an OPEN list and a CLOSED list. The OPEN list is a priority queue and it maintains the order of those nodes that have to be examined in near future, while the CLOSED list keeps track of those nodes that have already been examined.

Each node $n$ maintains $\mathrm{f}(\mathrm{n})$, where

$\mathbf{f}(\mathbf{n})=\mathbf{g}(\mathbf{n})+\mathbf{h}(\mathbf{n})$

which is the estimate of the best solution that goes through $\mathrm{n}$.

The heuristic function must be acceptable, i.e. it must not overestimate the distance to the goal or the estimated cost must be less than the actual cost. This produces computationally optimal results. Thus, for an application like routing, $\mathrm{h}(\mathrm{n})$ might correspond to the straight-line distance to the goal, since that is physically the smallest feasible distance between any two nodes. The most vital part of the A-Star algorithm is a good heuristic estimate function. This can enhance the efficiency and performance of the algorithm.

Here, in addition to $g(n)$ and $h(n)$, another parameter to measure traffic of a route has also been considered that keeps track of the current speed of the node. As introduced earlier, a pre defined threshold speed; Sp can be used as a threshold level for a node. If node's residual speed goes below this level, the algorithm should avoid this node while searching for a route (as an indicator of traffic on the open route), and instead it should search for alternative path, where no node having residual speed below $\mathrm{Sp}$ is there. This new parameter, 1(n) is the path cost count of weak node having less speed. This will keep a count on how many nodes in the current path are below speed of Sp.

Thus, Estimated cost function $\mathrm{f}(\mathrm{n})$ carries two parameters shown below:

$f(n)=(g(n)+h(n), l(n))$ 
In this approach, routing decision is made based on value of $1(n)$. If value of $1(n)$ is same then only, value of $f(n)$ is checked for further comparison.

\section{CONCLUSION}

VANETs are anticipated to prop a colossal spectrum of mobile distributed applications whose scope is from traffic alert dissemination and dynamic route planning to context-aware advertisement and file sharing. The performance of established routing protocols in VANET is very deprived. The main problem with these protocols is their Route Instability in VANET's. The established node-centric view of the routes (i.e., an established path is a fixed succession of nodes between the source and the destination) leads to frequent broken paths in the presence of VANETs' elevated mobility, Consequently, countless packets are dropped, and the overhead due to path repairs or failure notifications considerably increases, leading to low delivery ratios and elevated transmission delays.

\section{REFERENCES}

[1] Y.-C. Hu, A. Perrig, and D. B. Johnson, "Ariadne: A Secure On-Demand Routing Protocol for Ad Hoc Networks," Wireless Networks, vol. 11, no. 1-2, pp. 21-38, 2005.

[2] G. Jayakumar and G. Gopinath, "Ad Hoc Wireless Networks Routing Protocols - A Review," Journal of Computer Science, vol. 3, no. 8. pp. 574-582, 2007.

[3] R. Bruno, M. Conti, and E. Gregori, "Mesh Networks: Commodity Multihop Ad Hoc Networks," IEEE Communication Magazine, vol. 43, no. 3, pp. 123-131, 2005.

[4] W. Kiess and M. Mauve, "A survey on realworld implementations of mobile ad-hoc networks," Ad Hoc Networks, vol. 5, no. 3, pp. 324-339, 2007.

[5] A. Al Hanbali, E. Altman, and P. Nain, "A Survey of TCP over ad hoc networks," IEEE Communication Survey Tutorials, vol. 7, no. 3, pp. 22-36, 2005.

[6] F. Li, Y. Wang, and N. Carolina, "Routing in Vehicular Ad Hoc Networks : A Survey," Ieee Transportation Systems, vol. 7, no. 3, pp. 377-
Vehicular Technology Magazine, no. June, pp. 12-22, 2007.

[7] S. Sesay, Z. Yang, and J. He, "A survey on mobile ad hoc wireless network," Journal of Information Technology,vol. 3, no. 2, pp. 168$175,2004$.

[8] R. S. Raw, M. Kumar, and N. Singh, "Security Challenges, Issues and Their Solutions for VANET," International Journal of Network Security \& Its Applications, vol. 5, no. 5, pp. 95-105, 2013.

[9] S. Al-Sultan, M. M. Al-Doori, A. H. AlBayatti, and H. Zedan, "A comprehensive survey on vehicular Ad Hoc network," Journal of Network and Computer Applications, vol. 37, no. 1, pp. 380-392, 2014.

[10] S. Zeadally, R. Hunt, Y.-S. Chen, A. Irwin, and A. Hassan, "Vehicular ad hoc networks (VANETS): status, results, and challenges," Telecommunication Systems, pp. 217-241, 2010.

[11] V. Dahiya and A. Dureja, "Vehicular Ad Hoc network - Various Challenges and Applications," International Journal of Advancement in Engineering Technology, Management and Applied Science, pp. 45-54, 2014.

[12] K. Mehta, L. G. Malik, and P. Bajaj, "VANET: Challenges, Issues and Solutions," 2013 6th International Conference on Emerging Trends in Engineering Technology, pp. 78-79, 2013.

[13] P. I. Offor, "Vehicle Ad Hoc Network ( VANET ): Safety Benefits and Security Challenges," no. 2009, 2007.

[14] O. In, "TOPICS IN AD HOC AND SENSOR NETWORKS A Tutorial Survey on Vehicular Ad Hoc Networks," no. June, pp. 164-171, 2008.

[15] C. N. E. Anagnostopoulos, I. E. Anagnostopoulos, V. Loumos, and E. Kayafas, "A license plate-recognition algorithm for intelligent transportation system applications," IEEE Transactions on Intelligent 391, 2006. 
[16] C. Shen, W. Du, R. Atkinson, and K. H. Kwong, "Policy based mobility \& flow management for IPv6 heterogeneous wireless networks," Wireless Personal Communication, vol. 62, no. 2, pp. 329-361, 2012.

[17] F. Bai and B. Krishnamachari, "Exploiting the wisdom of the crowd: Localized, distributed information-centric VANETs," IEEE Communication Magazine, vol. 48, no. 5, pp.
138-146, 2010.

[18] N. L. Aung, H. M. Tun, Z. M. Naing, C. M. Nwe, and Z. M. Lwin, "Evaluation and Comparison of Performances between AODV and DSDV Routing Protocols for VANET at TIntersection," International Journal of Emerging Engineering Research and Technology, vol. 3, no. 6, pp. 38-48, 2015. 